(C) 2007 IPEM. Published by Elsevier Science Ltd. All rights reserved. Used with permission.

This paper was originally published in the Elsevier Science Journal, Medical Engineering \& Physics. Vol. 29, Issue 6, July 2007, p.677-690. Available at:

http://www.elsevier.com/locate/issn/13504533

doi: 10.1016/i.medengphy.2006.07.008

All associated graphics are separate files located in the CSUCI Institutional Repository

$\underline{\text { http://repository.library.csuci.edu }}$

\title{
Robust measures of three-dimensional vascular tortuosity based on the minimum curvature of approximating polynomial spline fits to the vessel mid-line.
}

Michael J. Johnson ${ }^{a}$ and Geoff Dougherty ${ }^{b, *}$

${ }^{a}$ Department of Mathematics and Computer Science, Faculty of Science, P.O. Box 5969, Safat 13060, Kuwait

b Applied Physics, California State University Channel Islands, Camarillo, CA 93012, U.S.A.

* Corresponding author. E-mail address: geoff.doughertv@csuci.edu 


\begin{abstract}
The clinical recognition of abnormal vascular tortuosity is important in the diagnosis of many diseases. This paper presents a novel approach to the quantification of vascular tortuosity, using robust metrics based on unit speed parameterizations of three-dimensional (3-D) curvature. The use of approximating polynomial spline-fitting obviates the need for arbitrary filtering of mid-line data which is necessary with other tortuosity indices. The metrics were tested using both two-dimensional and three-dimensional synthesized images that mimicked clinically significant pathologies: two of the three metrics were scale invariant, additive, and produced tortuosity values tailored to be independent of the resolution of the imaging system. Our methodology is designed to explicitly handle the challenge of noisy data, and is largely tolerant of the inaccuracies in the mid-line extraction. While all the proposed metrics are sensitive to gently curved vessels, the rms curvature of the smoothest path was more effective in recognizing abnormalities involving high-frequency coiling such as occurs in malignant tumors. We have also indicated how values from two projection images, such as acquired in biplane angiography, can be combined to give an accurate approximation of the 3-D value of this metric. Our proposed methodology is well-suited to automated detection and measurement, which are a prerequisite for clinical implementation.
\end{abstract}

Keywords: tortuosity; blood vessels; vessel mid-line; spline fitting. 


\section{Introduction}

Vascular tortuosity is the result of accumulation of curvature along blood vessel length. Sustained, elevated arterial tension eventually results in thinning of the medial vascular layer with consequent dilation and buckling of the affected vessels: tortuosity. Increased tortuosity often occurs in association with arteriosclerotic change in large, medium and small arteries; thus, a variety of vessels, ranging from the aorta to small retinal vessels, may be affected. The tortuosity of a vessel influences its flow haemodynamics and may exacerbate and compound the initial weakening of the vessel wall, leading to aneurysm formation and potential vascular rupture. While adventitial tethering of large vessels precludes significant twisting or torsion, the resultant curvature can be in different planes, so that a three-dimensional (3-D) measure of tortuosity is required.

Although vascular tortuosity is often estimated using a gross qualitative scale, e.g. mild, moderate, severe and extreme, there is a need to develop a quantitative measure of tortuosity that can be assessed automatically, in order to assess its severity and progression and to investigate the link between tortuosity and the evolution of disease processes [1-4]. Several possible measures have been proposed, but none has gained universal acceptance. They include the distance factor [3,5], the number of inflection points [4], the angle change along segments [6,7] and various line integrals of local curvature values $[4,8,9]$, which may be more conveniently computed from second differences of the vessel mid-line [10].

Vessel tortuosity does not have a formal clinical definition but there are clearly some intuitive properties, which a reasonable index must satisfy in order for it to correlate with the qualitative assessment of an expert observer. In order to obtain a clinically meaningful tortuosity measure, these properties need to be made explicit, and any proposed measure must 
be shown to satisfy them. The measure should be invariant to affine transformations of a vessel: translation, rotation and scaling. The position and orientation of a vessel do not alter the perception of tortuosity; nor should scaling, since the image of a vessel can be viewed at differing magnifications without affecting its perceived degree of tortuosity. The measure should be sensitive to the morphology of the vessel, i.e. to the shape of its path in space. The distance factor, the ratio of the meandering vessel length to the straight-line distance between its end points, is conceptually simple and easy to compute but does not satisfy this property. A vessel that bends gradually can yield the same numeric value of the distance index as one that bends more frequently, whereas what is required is that the more bends that occur along the length of a vessel, and the greater their amplitudes, the larger the tortuosity index should be. Most clinicians consider that an ideal tortuosity measure should be additive [8], i.e. the tortuosity of a composite vessel, comprising several portions, should equal the sum of the tortuosities of those portions. Some of the line integral measures [8] and the second difference index [10] satisfy these conditions. However, in their implementations, they are highly sensitive to noise, both from artefacts introduced during vessel extraction, to obtain the vessel mid-line, and to digitization errors, due to limited image resolution. The latter are compounded because of the use of small groups of consecutive, closely separated, discretely sampled data points to compute local curvatures.

Various low-pass filters have been applied to vessel mid-lines to mitigate digitization errors $[4,8,10]$, but these are ultimately arbitrary and affect the value of tortuosity obtained: the more severe the filtering, the smoother the mid-line and the lower the measured tortuosity. The use of cubic smoothing splines to filter noisy data [11] relies on an arbitrary weighting parameter and does not address the length of data sub-segment required.

This paper proposes the use of tortuosity metrics related to the curvature of a unit speed curve, obtained by approximating polynomial spline fitting to the discrete data points representing the mid-line of the vessel. Earlier metrics based on curvature $[4,8]$ were not well-defined because they used arbitrary parameterization; to avoid this problem 
reparameterization to a unit speed curve has been employed (Appendix A). Curvature based on the Frenet-Seret frame $[9,12,13]$ is undefined where the vessel is straight and at points of inflection, resulting in metrics whose values may fluctuate unpredictably for such examples. In the present method, the fitted curve is not required to pass through each mid-line data point, but rather approach it to within a distance related to the radius of the local blood vessel. The fitted curve is not restricted to the discrete pixel grid so that it can more closely correspond to the actual blood vessel. Approximating polynomial spline fitting captures the essential tortuosity of the vessels without having to place undue reliance on the accuracy of each extracted mid-line point, or employ arbitrary smoothing methods.

Analysis is construed directly in three dimensions (3-D) so that it can be applied to 3-D data sets, which are becoming increasingly available due to the thin, contiguous images now obtainable with helical computed tomography (HCT) and magnetic resonance angiography (MRA). Most previous work has focused on 2-D images, and very few analyses of tortuosity in 3-D have been attempted. A fundamental problem when working with 2-D projection images is that vessel crossings cannot be resolved. The 3-D variant of the distance factor [7, 14] is essentially a measure of vessel elongation rather than an indicator of vessel morphology. Methods based on counting the inflection points and the sum of angle changes have recently been extended to 3-D [9], with varying degrees of success. It should be noted that for 3-D data sets an estimation of torsion as well as tortuosity is required to completely define the morphology.

The utility of the proposed metrics in matching intuitive notions of tortuosity is investigated using computer-generated shapes of varying curvatures, with and without added noise, and simulated 2-D and 3-D blood vessels at differing resolutions. We demonstrate that our proposed metrics preserve scale invariance, additivity, and sensitivity to patterns of clinical significance when applied to noisy data. The relationship between the tortuosity of 3-D datasets and the 2-D projections of them is also explored. 


\section{Materials and Methods}

\subsection{Definitions and properties of metrics}

Morphologically, vessels are continuous structures. Let $d \boldsymbol{G}\{2,3,4, \ldots\}$ and let $f:[a, b] \rightarrow$ $\mathbf{R}^{\mathrm{d}}$ be a vector valued function such that $f, f$ and $f^{\prime \prime}$ are continuous. The function $f$ may be interpreted as tracing out the curve $C_{f}=\{f(t): a<t<b\}$ in $\mathrm{R}^{\mathrm{d}}$. It is held that $f$ is of unit speed if $\left.\mid f^{\prime}(t)\right\}=1$ for all $\mathrm{t}$. If $f$ is not of unit speed but satisfies the regularity condition $\left|f^{\prime}(t)\right|>0$ for all $t G[\mathrm{a}, \mathrm{b}]$, then it remains possible to reparameterize $f$, so that the resulting curve is of unit speed. Unit speed curves have the convenient feature that the unit tangent vector to the curve at $f(t)$ is given by $f(t)$ and the curvature by $f^{\prime \prime}(t) \backslash$. Moreover, any quantity that is defined on a unit speed function $f$ will depend solely on the geometric curve, rather than on the particular choice of parameterization. The length of $f$, denoted $L(f)$, is given by:

$$
L(f)=b \mid f^{\prime}(t) \backslash d t=b \quad 1 d t=b-a
$$

where the integrand has simplified, since $f$ is of unit speed.

The mean curvature, denoted $M(f)$, is defined by:

$$
\left.M(f)={ }_{a}^{b} \Gamma f(t)\right\rfloor d t
$$

and the mean square curvature, denoted $J(f)$, is defined by:

$$
J(f)=\stackrel{b}{\mathbf{I}} \mid f(t) \backslash d t
$$


The essential difference between $M(f)$ and $J(f)$ is that the former is a democratic integral of the curvature, i.e. all points on the curve are equally weighted, whereas the latter gives more weight when the curvature is higher.

The normalized root-mean-square (rms) curvature of a unit speed curve, denoted $K(f)$, is defined by:

$$
K(f)=y J L(f) . J(f)=(b-a) b \backslash f(t) \backslash^{2} d t
$$

$M(f)$ and $J(f)$ represent, respectively, analogs of Hart's metrics $\mathbf{T}_{4}, \mathrm{~T}_{6}$ and $\mathrm{T}_{5}, \mathrm{~T}_{7}[8]$, except that these latter metrics were not defined in terms of unit speed curves.

The metrics $M(f)$ and $K(f)$ are scale invariant, but $J(f)$ is not (Appendix B). The property of scale invariance is considered highly desirable for a tortuosity metric, since its value would then be independent of the image size. Most ophthalmologists, who are experienced at grading narrow tortuous vessels, intuitively believe that tortuosity should be relatively independent of scaling [8]. Tortuosity metrics that are not dimensionless, e.g. the tortuosity index of [13], are not scale invariant.

The property of additivity is another important consideration for a tortuosity metric. It is considered that if a curve is partitioned into two sub-curves, then the total tortuosity should equal the sum of the tortuosities of the sub-curves. $M(f)$ and $J(f)$ are clearly additive from their definitions, and $K(f)$ is additive when $J(f) / L(f)$ takes the same value for each sub-curve (Appendix C). This is in contradistinction to Hart et al's metrics [8], where the tortuosity does not increase if the same pattern is extended to greater lengths. If unit speed parameterization were used with Hart et al's metrics, then their total curvature tc(C) would 
correspond with the above mean curvature $M$, and their total square curvature $\operatorname{tsc}(\mathrm{C})$ would correspond with the above mean square curvature $J$; they did not consider a metric similar to the present paper's normalized root-mean-square curvature $K$. Hart et al's response to scaling is flawed because rescaling the curve requires a choice of parameterization, and this choice affects the values obtained for the metrics.

It is possible to extend the definitions of $M(f), J(f)$ and $K(f)$ to unit speed piece-wise linear functions (Appendix D). The connecting corners can be replaced by arcs of circles of very small radii.

In medical imaging, instead of having an analytic expression for a given curve, one has a sequence of sampled points, obtained from the mid-line of a vessel that is close to the idealized curve, with the resultant problem of approximating the values of $M(f), J(f)$ and $K(f)$ for a curve based only on such imprecise data. Tortuosity metrics tend to be rather sensitive to the presence of noise, e.g. due to digitization errors, largely as a consequence of the appearance of the second derivative of $f$ in their defining integrals. In order to get a meaningful estimate of their values it is essential that a smoothing process be applied to the data, so as to reduce the effects of noise to a tolerable level. However, the amount of smoothing performed will have a significant effect on the estimates obtained; it is crucial to have a systematic smoothing technique whose degree of smoothing can be controlled to such an extent that its effect on the estimated values will be acceptably independent of the resolution of the data, and hence of the imaging system used to acquire it.

For real (noisy) data it can be shown that there exists unit speed piece-wise linear functions, which pass through 'data balls' of radius $r_{i}$, centered on the points, $x_{i}$, defining the mid-line, 
and which minimize $L(f)$ and are unique (Appendix E). The radii of the data balls can be specified in terms of the local radius of the vessel, $\mathrm{R}_{\mathbf{i}}$. This 'shortest path', $f_{l}$, between the data balls (Appendix F) is used to define the mean curvature, $M(f)$. Once this has been obtained, an algorithm is used to find the 'smoothest path', $f_{2}$, connecting these points, and hence to calculate $J(f)$ and $K(f)$. The 'smoothest path' is also expected to be unique, although this has not been proven (Appendix F). An interesting feature of the present metrics is that they do not require the sampled points to be regularly spaced.

The functionals $M(f), J(f)$ and $K(f)$ are all putative tortuosity metrics, although $J(f)$ may be less desirable because it is not scale invariant. The smoothing process has to be chosen with care in order to maintain the properties of the underlying curve functional. We have chosen the shortest path through the data balls as the smoothing process for the curve functional $M(f)$, and the smoothest path through the data balls as the smoothing process for $K(f$ (and $J(f)$ ). These smoothing processes have been carefully tailored for their underlying curve functionals. In particular, scale invariance is preserved mathematically and additivity is reasonably preserved. It is not computationally practical to find the smoothest curve by minimizing the value of $M(f)$ over all curves passing through the data balls, but we have adapted Kersey's near interpolation method $[15,16]$ to approximately compute the smoothest

path. In doing so, we avoid the arbitrariness of choosing a weighting parameter and subsegment length, which are necessary in using smoothing splines.

\subsection{Test images}

A difficulty in defining medically useful measures of tortuosity is that the clinical definition of 'abnormal tortuosity' can vary from disease to disease. Three distinct patterns of abnormal 3-D vascular tortuosity, each associated with differing disease processes, have been defined [9]. Type I abnormalities occur when a normally straight or gently curved vessel begins to exhibit a more sinuous curve, i.e. of relatively low frequency and large amplitude. This is characteristic of the changes that occur in vessels with aging, hypertension, atherosclerosis or 
retinal disease of prematurity. Type II abnormalities involve tightly packed clusters of vessels that exhibit frequent and unpredictable changes of direction; they are of variable length, with medium frequencies and amplitudes, and are typical of vessels within highly vascularized tumors and within the nidi of arteriovenous malformations (AVMs). Type III abnormalities exhibit high frequency (tight) coils or sinusoids of low amplitude and are typical of the vessels in malignant brain tumors. Any clinically useful tortuosity metric needs to be able to characterize patterns of morphological abnormality such as these to the satisfaction of an experienced clinician.

(i) Helices were generated using the parametric equation:

$$
p(t)=[a \cos (t), a \sin (t), b t]
$$

where ' $a$ ' is the radius and ' $b$ ' is the pitch. The theoretical curvature $(x)$ of a helix is given by: $a /\left(a^{2}+b^{2}\right)[17]$. Various values of $a, b$ and $N$, the number of helical turns, were used. The two degenerate cases of a helix are a circle $(b=0)$ with maximum curvature, and a straight line in the $\mathrm{z}$-direction $(\mathrm{a}=0)$ with minimum curvature. When the pitch $(\mathrm{b})$ is zero, the helix becomes a circle with radius a, and maximal curvature (=1/a). As the pitch (b) is increased it becomes a helix, and then approaches a straight line (as $b>>a$ ). Additionally, a composite helix was simulated, comprising one turn each of three different pitches, arranged so that the three turns connected smoothly with each other. A data ball size, i.e. radius, (r) of 0.001 was used in the computation of the shortest and smoothest paths, and hence of $M(f), J(f)$ and $K(f)$.

'Noisy' helices were simulated by adding random components, in all three directions, from a normal distribution, with various magnitudes relative to the radius of the helix (with $\mathrm{a}=10, \mathrm{~b}$ $=10, \mathrm{~N}=2$ ); the amount of added noise was characterized by a signal-to-noise ratio (SNR) which varied from 20 to $58 \mathrm{~dB}$. Fig. 1 shows a 'noisy' helix with a SNR of $34 \mathrm{~dB}$. Data ball sizes of $0.01,0.025,0.1$ and 0.25 were used to compute the shortest and smoothest paths. 
(ii) A 2-D image mimicking a blood vessel was synthesized using fractional Brownian motion in the y-direction, to define a mid-line, with two roughly equidistant lines on either side defining the vessel boundaries. The nominal radius of the synthesized vessel, $R_{\text {nom }}$, was 0.07. Its boundaries were delineated by square pixels of edge length $h$, for values of $h$ from 0.001 to 0.2 , by superimposing different grids over the vessel and noting the active pixels; this produced images at differing resolutions to mimic images acquired at varying resolutions from different imaging systems. The pixel centers were taken as the boundary points to be fitted to within the chosen data ball size. The simulations were run with various data ball sizes, $r$, related to the local radius of the vessel, $\mathrm{Ri}$ (viz., $r=\mathrm{Ri} / 10, \mathrm{R}_{\mathrm{i}} / 4$ and $\mathrm{Ri} / 2$ ).

In a further set of simulations, the precise location of each boundary point was allowed to vary within its pixel, a process that will be referred to as shortest path pixel rectification (SPPR), so as to minimize the shortest path. In order that $M$ and $K$ not significantly decrease as $\mathrm{h}$ increases, which would be undesirable, the boundary points were constrained to vary within the sub-pixel, having the same centre but half the edge length; this will be referred to as 50\% SPPR. This constraint is analogous to using data balls, except that in place of balls squares are used, i.e. pixels.

(iii) It is instructive to know, at least statistically, how the projection of a 3-D curve on to a 2-D plane affects the measured tortuosity values. We used parametric curves based on integrated Brownian motion (Appendix G). Four thousand 3-D curves, which were loosely tied to the $x y$-plane with an angle between the unit tangent vector and the $x y$-plane, $(\mathbf{p}$, restrained to be no more than 50 , and variances $\mathrm{o}_{\mathrm{i}}^{2}$ chosen randomly with $0.05<\mathrm{o}_{\mathrm{i}}^{2}<0.2$. (This mimics the situation in the retina, for example, where the tangent vectors of the retinal 
vessels are nearly parallel to the plane of the thin choroid which contains them). With 250 nodes and a time step, At, of 0.04 the expected curve length was 10 .

Further 3-D curves were synthesized which were loosely tied to the $z$-axis with the angle between the unit tangent vector and the $x y$-plane restrained to be at least $\mathbf{c p}$ and variances $\mathrm{o}_{i}^{2}$ chosen randomly with $0.05<\mathrm{o}_{\mathrm{i}}^{2}<0.2$ (Appendix $\mathrm{G}$ ). Given the projections $\mathrm{Cxz}$ and $\mathrm{C}_{\mathrm{yz}}$ of $\mathrm{C}$ on to the $x z$ - and $y z$-planes respectively, we can compute the 2-D tortuosity values. Our task is to combine them to predict the 3-D values of tortuosity. This mimics the problem of trying to obtain the 3-D tortuosity of a vessel directly from measurements on biplane angiograms.

\subsection{Image analysis}

The mean curvature $M(f)$ was computed from the shortest path using unit speed linear segments connecting the data, with or without data balls. A smooth piece-wise cubic spline, i.e. with the minimum rms curvature, passing through all the data balls, of a specified size, was then fitted iteratively, starting from these unit speed linear segments, and used to compute $K(f)$ (and $J(f)$ ).

All routines were written in GNU Octave running under the Linux operating system on a Pentium IV PC. The bulk of the computational cost in computing the metrics is in computing the shortest and smoothest paths. These paths are computed iteratively with fixed point iteration. The total computational cost is roughly proportional to the number of data balls.

\section{Results}

The methodology was tested in 3-D, by the manipulation of the variables of simple helices Table 1 shows the theoretical curvatures, and the measured values of the lengths of the helices (L) and of the functionals $M, J$ and $K$. The measured lengths of the helices agreed 
with the theoretical length of $2 \Pi \hat{j} a^{2}+b 2$. (The values for $2-\mathrm{D}$ projections, on to the coronal (Y-Z) and sagittal (X-Z) planes, of the data points were also computed). For the 'noisy' helices, the computed values of $M$ and $K$ for different data ball sizes were shown as a function of SNR (Fig.2).

The smoothest path through the synthesized 2-D blood vessel, using a data ball size equal to the nominal radius of the vessel $\left(\mathrm{R}_{\mathrm{i}}\right)$, is shown in Fig.3(a): in this situation the smoothest path stays only just within the vessel. In practice, smaller data ball sizes should be used to constrain the smoothest path to a more central position: Fig.3(b) shows the smoothest path through the same vessel using a data ball size of $\mathrm{Ri} / 4$.

The values of the metrics $M$ and $K$ obtained for the 2-D vessel at various resolutions, characterized by the respective pixel size, h, are shown in Fig. 4, for selected data ball sizes $(\mathrm{Ri} / 10, \mathrm{Ri} / 4$ and $\mathrm{Ri} / 2)$. The values obtained at the highest resolution $(\mathrm{h}=0.001)$ were taken to be the optimum values, and the first value of $\mathrm{h}$, for which $M$ (or $K$ ) deviated by more than $10 \%$ from this value, was recorded. These values indicate how low the resolution of the image can drop and still result in an acceptable value of $M($ or $K)$. The computed $M$ and $K$ values show considerable variation since the precise path lengths, $L_{1}$ and $L_{2}$, change during each simulation; in order to minimize this effect the data was smoothed using a zero-phase five point running average filter. Fig. 5 shows the values of $\mathrm{M}$ and $\mathrm{K}$ under equivalent conditions to Fig. 4, but using 50\% SPPR.

As an alternative strategy to reduce the variation in the $\mathrm{M}, \mathrm{K}$ values 100 different vessels were synthesized at selected resolution (h) values for the selected data ball sizes $\left(\mathrm{Ri} / 10, \mathrm{R}_{\mathrm{i}} / 4\right.$ and $\mathrm{R}_{1} / 2$ ). From these trials the resolution value (h) was identified, which resulted in a $95 \%$ 
success rate, i.e. $95 \%$ of the values of $\mathrm{M}$ and $\mathrm{K}$ were predicted to be within $10 \%$ of the optimum value.

For 3D synthesized parametric curves based on integrated Brownian motion and tied to the xy-plane by $\left(\mathbf{p}<50\right.$ the ratios of $M_{2 \mathrm{D}} / M, J_{2 \mathrm{D}} / J$ and $K_{2} D / K$ were $0.665( \pm 21 \%), 0.549( \pm 38 \%)$ and $0.735( \pm 21 \%)$.

The strategy for combining the 2-D tortuosity values from projections on the $x z$ - and $y z$ planes to give estimates of the 3-D tortuosity values is explained in Appendix G. Table 2 shows how close these estimates are to the true 3-D tortuosity values for synthesized curves which are loosely tied to the $z$-axis by angles of at least ( $\mathbf{p}$.

\section{Discussion}

Table 1 shows that each of the functionals $M, J$ and $K$ can discriminate between the different helices, correctly assigning the highest values to the tightest coils. However, they differ from

the theoretical curvature, $\mathbf{K}$, in that their values are proportional to the path lengths $\left(L 1\right.$ or $\left.L_{2}\right)$ measured along the curve, whereas $\mathbf{K}$ is independent of length; this is a consequence of the additivity property built into them. (Should a length-independent metric be required, then the length-normalized variants $\left(M L 1, J / L_{2}\right.$ and $\left.K L_{2}\right)$ must be used; interestingly, the numerical values of $K L_{2}$ for smooth helices are equal to the values of the theoretical curvature, $\mathrm{K}$ ). When the radius and pitch are both doubled the values of $M$ and $K$ remain unchanged, confirming that they are scale invariant; the theoretical curvature, $\mathbf{K}$, on the other hand, is not scale invariant, and neither is $J$. 
With a composite helix, comprising three turns of differing pitch joined smoothly, the $M, J$ and $K$ values for each turn are added, whereas the individual values of $x$ and the normalized metrics $\left(M / L_{1}, J / L_{2}\right.$ and $\left.K / L_{2}\right)$ are averaged. A numerically identical value of $x$ (and $K / L_{2}$ ) can be obtained from a uniform helix by adjusting its pitch. This demonstrates the deficiency of a global metric such as $x$ or $K / L_{2}$, or indeed any of the metrics, uniquely to characterize the curvature of a vessel; in order to distinguish variations in local curvature, local tortuosity values would need to be computed and displayed, for example, in histogram form[12].

The estimates of $M$ and $K$ are large when there is a lot of noise, but decrease as the noise is reduced, i.e. larger SNR; this is a result of the shorter and smoother paths available with progressively smaller amounts of noise. This behavior is demonstrated in Fig.2, where the estimates of $M$ and $K$ for the noisy helices approach the values for the smooth helix at SNRs of $40-55 \mathrm{~dB}$, depending on the data ball size. This asymptotic approach occurs at even smaller SNRs when a larger data ball size is chosen. The smoothest path is akin to an active contour (or snake), which adjusts its curvature in the light of the constraints operating: with a larger data ball size the smoothest path can become straighter. Most digital radiological images, except those in nuclear medicine, will have SNRs around $60 \mathrm{~dB}$ or above, as a consequence of digitization with an ADC of 10 bits or more [18]. 3-D contrast-enhanced MRA images have SNRs around $30 \mathrm{~dB}$ [19], and ophthalmoscope images have lower SNRs ( $\sim 16 \mathrm{~dB})[20]$ although these values can be increased significantly by averaging images [21].

Noise in positional data propagates with the derivative, so that higher-order derivatives suffer larger variance when noise is present. Tortuosity metrics depend on the second derivative and are thus highly sensitive to noise. Variance in the curvature increases as the distance separating sampled points decreases, and this has been a major limitation in previous 
tortuosity algorithms $[4,8,10]$ that have used the coordinates of sampled points along the mid-line directly. The present scheme, of using polynomial spline fitting, minimizes this effect, although it will eventually become significant for vessels from images acquired at very low signal-to-noise ratios. The torsion experienced by blood vessels depends on the third derivative of the curve, and thus is even more susceptible to noise, particularly for vessels that are almost straight. Torsion is considered to be more relevant to flow measurements, and as such, its analysis has not been included in the present study.

It is important to know whether or not the tortuosity metrics depend on the resolution of the image from which they are calculated. Fig. 4 shows examples of the variation in computed values of $M$ and $K$ for a range of resolutions, characterized by the pixel size, h, using different data ball sizes $\left(\mathrm{R}_{\mathrm{i}} / 2, \mathrm{R}_{\mathrm{i}} / 4\right.$ and $\left.\mathrm{R}_{\mathrm{i}} / 10\right)$. Fig. $4(\mathrm{a})$ indicates that the values of $M$ and $K$ remain within $10 \%$ of their optimum values, i.e. those measured at the highest resolution ( $h=0.001$ ), up to values of $\mathrm{h}=0.045$ and 0.055 respectively, corresponding to pixel sizes of $64-79 \%$ of the vessel radius, i.e. 0.07 , when a large data ball size of $\mathrm{R}_{\mathrm{i}} / 2$ is used. For smaller data ball sizes $\left(\mathrm{R}_{\mathrm{i}} / 4\right.$, Fig. 4 (b)), corresponding to tighter limits on the fitting, constant values of $M$ and $K$ are maintained within $10 \%$ of their optimum values, but not to such low resolutions, viz. to pixel sizes of $51-53 \%$ of the vessel radius; and for even smaller data ball sizes $\left(\mathrm{R}_{\mathbf{i}} / 10\right.$, Fig. $\left.4(\mathrm{c})\right)$, constant values of $M$ and $K$ are maintained only up to pixel sizes of $26-30 \%$ of the vessel radius. Using multiple simulations at selected resolutions, the corresponding values for a $95 \%$ success rate, i.e. $95 \%$ of the values of $M$ and $K$ within $10 \%$ of the optimum value, were a little lower: $50 \%, 34 \%$ and $24 \%$ of vessel radius for data ball sizes of $R_{i} / 2, R_{i} / 4$ and $R_{i} / 10$ respectively. 
If $50 \%$ SPPR is used on the boundary data prior to using data balls, the values of $M$ and $K$ remain constant, within $10 \%$, at all practical resolutions, down to where the pixel size is similar to the radius of the vessel, $h=0.07$ ( Fig. 5). During digitization of the synthesized vessel the boundaries of the vessel will at times pass close to the border between two pixels, and the digitized boundaries must choose one or other pixel, introducing artifacts, which increase the computed values of $M$ and $K$. The $50 \%$ SPPR method is effective at smoothing out this effect. Furthermore, at low resolutions, i.e. where the pixel size approaches the radius of the vessel, the digitized right and left boundaries may intersect; the 50\% SPPR reduces the likelihood of this happening. The SPPR pre-processing method is still somewhat experimental and the $50 \%$ fraction is ad hoc; although it delivers tortuosity values independent of resolution, its use complicates the algorithm for computing $\mathrm{M}$ and $\mathrm{K}$ and hence its use cannot be recommended without reservation in all cases. If the image resolution is reasonably good, i.e. pixel size less than or about one-third of the vessel radius, there is no need for it, if the fitting is done with data ball sizes of one-quarter of the vessel radius or larger.

Our simulations with integrated Brownian motion curves show that it is impossible to accurately calculate the $3-D$ values of $M, J$ or $K$ given only $C_{x y}$, the projection of the 3-D curve on to the $x y$-plane. However if two projections are available, the problem becomes more tractable. Table 2 shows that, if the 2-D tortuosities are combined appropriately to give $J(0$ and $K(C)$ then the predicted values of $J$ and $K$ are relatively close to the actual (3D) values of $J$ and $K$ (where the relative distances, $\operatorname{dist}_{\mathrm{r}}(J, J)$ and $\operatorname{dist}_{\mathrm{r}}(K, K)$ are defined in Appendix G). This is especially true when the tangent vectors to the curves are constrained to lie within 200 of the $z$-axis, in which case our predicted values of $\mathrm{J}$ and $\mathrm{K}$ are likely to be

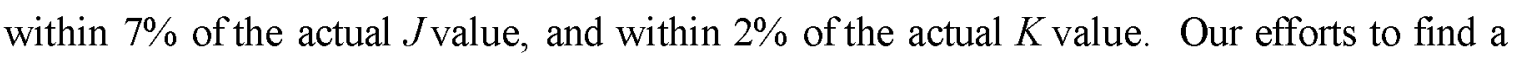
method of combining the 2-D values of $M$ into 3-D values have so far been confounded by the square root in the definition of $M$. 
If a tortuosity metric is to be useful in detecting and characterizing abnormal patterns of tortuosity, it should give higher tortuosity values to curves with higher frequencies and higher amplitudes. Families of sinusoids and helices can be used to illustrate type I and type III abnormalities in 2-D and 3-D data sets respectively [9], with type II abnormalities being a hybrid. Sinusoids of the form $y=1 / n \cdot \sin (n x)$, where $n$ is a positive integer and $0<x<2 \%$, illustrate type I abnormalities in 2-D. It is generally accepted that the tortuosity should increase as $\mathrm{n}$ increases. (The distance factor does not satisfy this property, illustrating its shortcomings as a tortuosity metric). It can be shown that, for a noiseless sinusoid, $M=7 \mathrm{~m}$ and $K=3.98 \mathrm{n}$ (and $J=2.07 \mathrm{n}^{2}$ ). Helices of the form $\mathrm{x}=1 / \mathrm{n} \cdot \cos (\mathrm{nt}), \mathrm{y}=1 / \mathrm{n} . \sin (\mathrm{nt}), \mathrm{z}=\mathrm{t}$; $0<\mathrm{t}<2 \%$ illustrate type I abnormalities in 3-D: such helices, in the absence of noise, result in $M$ and $K$ values of V2.7n (and $J=\% n^{2} / \mathrm{V} 2$ ). Since helices have constant curvature, $M$ (the mean curvature) and $K$ (the rms curvature) are equal; sinusoids have a variety of curvatures so that $M £ K$. Thus $M$ and $K$ (and even $J$ ) are potentially useful for investigating type I abnormalities in both 2-D and 3-D data sets, since their values increase as $\mathrm{n}$ increases.

Type III abnormalities in 2-D can be illustrated by the family of sinusoids $y=a \sin x, 0<x<$ $2 \%$ where a $>0$ denotes the amplitude of the sinusoid. It is expected that the value of a useful tortuosity metric should increase as the amplitude, a, increases. It can be shown analytically for this case that $M=4 \tan ^{-1}$ a. Although $M$ increases with amplitude initially, it is bounded above by $2 \mathrm{rc}$, indicating that it is somewhat insensitive to type III abnormalities. It is not possible to obtain analytic expressions for $K$ and $J$. Instead numerical values were obtained and are shown plotted in Fig. 6(a). While $M$ is sensitive to type III abnormalities only when the amplitude is small, the distance factor and $J$ are sensitive only at large amplitudes. $K$, however, is sensitive at all amplitudes. 
Since tortuosity should be scale invariant, the tortuosity of circular helices should not increase without bound as their radius increases as proposed earlier [9]. We consider that elliptic helices of the form $\mathrm{x}=\mathrm{a} \cos (\mathrm{t}), \mathrm{y}=\sin (\mathrm{t}), \mathrm{z}=\mathrm{t} ; \quad 0<\mathrm{t}<2 \%$ are more appropriate at illustrating type III abnormalities. For $0<\mathrm{a}<10$, we numerically computed the values of $M$, $K$ and $J$; note that we are primarily interested in values of a $>1$. For a $>1, M$ is rather insensitive to type III abnormalities, while $K$ and $J$ (and the distance factor) are sensitive (Fig. 6(b)). Thus, to summarize, $M$ and $K$ have similar sensitivities to type I abnormalities, whereas $J$ scales as the square of the frequency which does not match intuitive notions of tortuosity: with type III abnormalities $K$ and $J$ are sensitive, but $M$ is insensitive.

The considerable range over which the computed values of $M$ and $K$ are independent of image resolution adds to the clinical usefulness of these metrics. The present results indicate that using a data ball size of one-quarter of the local vessel radius may be suitable for most vascular systems, since it offers accurate fitting to the vessel mid-line and allows the use of relatively low-resolution images if better images are not available. For wide, nearly straight even more accurate fitting of the polynomial splines to the mid-line may be desirable, in which case a data ball size of, say, one-tenth of the local vessel radius would be more appropriate, and better resolution images, with a pixel size less than $25 \%$ of the vessel radius, would be required. $K$ is the more effective metric for quantifying tightly wound vessels.

We have defined computationally tractable metrics, construed in 3-D but applicable to 2-D datasets also, and rigorously proved their scale invariance and additivity properties. Our methods are designed to explicitly handle the challenge of noisy data, resulting from random noise or digitization errors, without invoking arbitrary smoothing which would compromise 
these characteristic properties. They are largely independent of the accuracy of the mid-line extraction process, so that relatively unsophisticated algorithms could be used for this preprocessing stage. Different metrics are able to detect different abnormalities, but $K$, the rms curvature of the smoothest point through the mid-line data points, is the most effective at evaluating the range of clinically relevant patterns of abnormality.

We are applying these metrics to a number of clinical vascular systems, using 3-D data sets from $\mathrm{HCT}$ and MRA, in order to test their clinical utility in quantifying the severity of stenoses and aneurysms. Preliminary results indicate that they are largely tolerant of inaccuracies in the definition of vessel walls and subsequent mid-line extraction, and are sufficiently discriminating to assess the relative utility of arteries for endoluminal repair. They may also be able to distinguish several different pathological conditions in retinal vessels. Our metrics, in common with earlier proposals, do not incorporate torsion of the vessels. This would involve the use of third derivatives, and consequent problems of instability (as the vessel becomes straight) and hypersensitivity to inaccuracies in the data. Our results to date indicate that many practical systems can be analyzed without resorting to such additional sophistication.

The tortuosity values that have been computed thus far apply to vessel segments. Current work is concerned with generalizing this treatment, in order to characterize a network of branched vessels, such as occur in the microvascular network of the retina. An earlier attempt at this [22] used the average tortuosity of vessel segments to characterize such a network.

Acknowledgements 
We are grateful to Dr. Scott Kersey for his comments and suggestions regarding the "smoothest path" algorithm.

\section{Appendix A}

For a given curve $\mathrm{C}=(x(t), y(t))$, te $\left[t_{0}, t_{1}\right]$, Hart et al [8] define arc length $\mathrm{s}(\mathrm{C})$, chord length $(\mathrm{C})$, total curvature $\operatorname{tc}(\mathrm{C})$ and total squared curvature tsc(C). While arc length and chord length are independent of parameterization, total curvature and total squared curvature are not. To illustrate this, consider two different parameterizations for the parabolic curve: $y=x^{2}, 0<x<1$

(i) With the parameterization $x(t)=t, y=t^{2}, 0<t<1$, the curvature $\mathrm{K}(t)=2 /(1+$ $\left.4 t^{2}\right)^{3 / 2}$ and integration gives tc $(C) \sim 0.894427$ and $\operatorname{tsc}(C) \sim 1.17036$.

(ii) With the parameterization $x(t)=t^{2}, y=t^{4}, 0<t<1, K(t)=2 /\left(1+4 t^{2}\right)^{3 / 2}$ and integration gives tc $(C) \sim 1.26960$ and $\operatorname{tsc}(C) \sim 2.05820$.

The arc length and chord length of the curve, regardless of parameterization, are $\mathrm{s}(\mathrm{C}) \sim$ 1.47894 and chord(C) 1.41421.

It follows that their tortuosity metric $\mathbf{i}_{1}$ (the distance factor) is independent of parameterization, but the others $\left(\mathrm{x}_{2}, \mathrm{x}_{3}, \ldots ., \mathrm{x}_{7}\right)$ depend on the parameterization.

Appendix B 
It is important to understand how the functionals $L(f), M(f)$, and $J(f)$ behave when the curve is scaled. Let $A>0$ be a scaling parameter and define $\mathrm{f}_{x}:[\wedge a, A \mathrm{~b}] \rightarrow \mathrm{R}^{\mathrm{d}}$ by:

$$
f_{x}(t)=A \cdot f(t / A)
$$

The curve $\mathrm{C}_{\mathrm{A}}$ equals the curve $C_{f}$ scaled by $x$ in the sense that $\mathrm{x} e C_{f}$ if and only if Axe $C f l$. Note that $f^{\wedge}$ is also of unit speed because

$$
f()=\mathrm{A} \frac{-}{d t} f(t / A)=f^{\prime}(A t) \quad 1
$$

It is clear that $L\left(f_{x}\right)=A L(f)$. For the mean curvature, it can be seen that

$$
M\left(f_{x}\right)=\mathbf{f} \quad A^{x b} d d^{2} f(t / A) d t={ }^{b} \longrightarrow d t={ }^{b}\left|f^{f f}(\mathrm{r})\right| \quad d r=M(f)
$$

so that $M\left(f_{x}\right)=M(f)$. Thus the mean curvature is scale invariant. As for the mean square curvature,

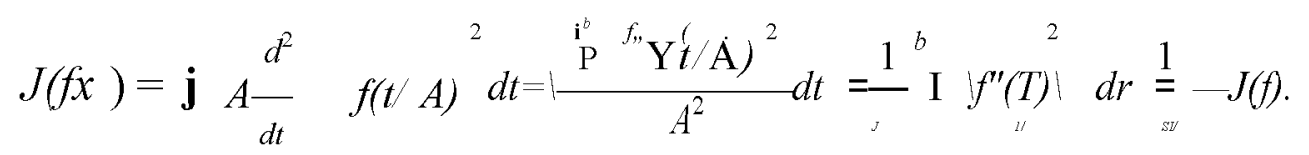

Thus the mean square curvature has the scaling property $J(A f)=1 / k J(f)$ and is consequently not scale invariant. The normalized root mean square (rms) curvature, denoted $K(f)$ is defined by:

$$
K(f)=\cdot j L(f) J(f)
$$

Note that $\quad K(f x)=\wedge L\left(f_{x}\right) J\left(f_{x}\right)=\wedge A L(f) 1 / A J(f)=K(f)$, and hence the normalized rms curvature is scale invariant.

Appendix C 
Consider the situation when the curve $f:[a, b] \rightarrow \boldsymbol{R}^{d}$ is partitioned into two sub-curves $f_{1}$ : $[a, c] \rightarrow \boldsymbol{R}^{d}$ and $f_{2}:[c, b] \rightarrow \boldsymbol{R}^{d}$ with $f_{1}(t)=f(t), t[a, c]$ and $f_{2}(t)=f(t), t[c, b]$ We seek to derive relations between $M(f)$ and the pair $M(f 1), M\left(f_{2}\right)$, and similarly between $K(f)$ and the pair $K(f 1), K\left(f_{2}\right)$. It is clear from their definitions that $L, M$ and $J$ are additive: that is, $L(f)=L(f 1)+L\left(f_{2}\right), M(f)=M(f 1)+M\left(f_{2}\right)$ and $J(f)=J(f 1)+J\left(f_{2}\right)$. It turns out that $K$ is additive in the special case, when $K(f 1) / L(f 1)=K(f 2) / L(f 2)=p$; thus we will refer to $K$ as being conditionally additive. Indeed,

$\left.K(f)=s J L(f) J(f)=y j L(f)\left(J\left(f_{1}\right)+J^{\prime}\right)\right)={ }^{\wedge} L(f)(\rho L(f 1)+\rho L(f 2))$

$$
=\quad L(f) \boldsymbol{y} \rho=L(f 1) \boldsymbol{y} \rho+L\left(f_{2}\right) \boldsymbol{y} \rho=K(f 1)+K\left(f_{2}\right) .
$$

Thus for the general case:

$$
\begin{aligned}
& K(f)=-y J L(f) J(f)={ }^{\wedge} L(f)\left(J\left(f_{1}\right)+J\left(f_{2}\right)\right) \\
& =\quad M \frac{L(f)}{L(f 1)} K\left(f_{1}\right)+{\stackrel{L(f)_{f}}{L(f 2)}}^{K\left(f_{2}\right)} \\
& =\begin{array}{ll}
L(f) & L(f) \\
L\left(f_{1}\right) & L\left(f_{2}\right) \backslash L(f)^{\underline{2}} K\left(f_{1}\right)+L\left(f_{L\left(f^{1}\right)}\right) K\left(f_{2}\right)
\end{array}
\end{aligned}
$$

It follows from this that:

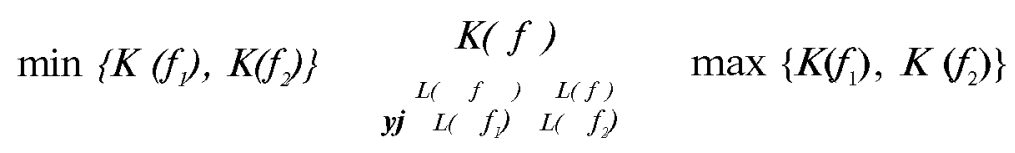

where the number dividing $K(f)$ is $>2$ and equals 2 if and only if $L(f 1)=L\left(f_{2}\right)=1 / 2 L(f)$ 


\section{Appendix D}

A function $f:[a, b] \rightarrow{ }^{d}$ is a unit speed piece-wise linear function if there exists $a=t_{1}<t_{2}$ $<\ldots<n=b$ such that:

$$
t i \quad-t=1 f\left(t_{i} \quad 1\right)-f(t i)
$$

and

$$
f(t)=\underset{t_{i}}{\underbrace{1}_{+1}-t_{i}} f\left(t_{i}\right)+\underset{t_{i+1}-t i}{f(}\left(t_{i}+1 \quad\right), \quad t G\left[t_{i}, t_{i}+1\right], \quad \text { (D.I) }
$$

for $i=1,2, \ldots ., n-1$.

For such a function $f$,

$$
\stackrel{\dot{2}}{f(t)}=\underset{f\left(t+{ }_{1}\right)-f(t)}{t i+1-t i} \text { whenever } t^{\wedge}\left(t_{i}, t i+1\right)
$$

Thus $f^{\prime}$ is a constant unit vector on each interval $(t, t i+1)$ and has, possible, jump discontinuities at the points $t_{2}, t_{3}, \ldots . . t n-1$. It follows that fis of unit speed in the sense of $2 \nmid f(t) \quad l d t \quad=t_{2}-t_{1} \quad$ whenever $\quad a<t_{1} \quad<\quad t_{2}<b \quad$ (D.2)

and if $f$ does indeed have jump discontinuities, i.e. if the minimal choice of $n$ is greater than 2 , then $f^{\prime \prime}$ is not a function and so the original definition of $M(f)$ cannot be used. What can be done is to replace the corners in the curve $\mathrm{C} f$ with arcs of circles having very small radii and to compute the mean curvature of the resultant curve.

Assume that $n$ is minimal so that $f$ has jump discontinuities at $t_{2}, t_{3}, \ldots . ., t_{n}-1$. Given a very small $\boldsymbol{s}>0$, define the curve $\mathrm{C}_{\mathrm{fe}}$ as follows: let $\mathrm{C}_{\mathrm{fe}}$ be the same as $\mathrm{C} f$, except near the points $\{f(t i)\} n={ }_{2}^{-}$. Near these points, replace $\mathrm{C} f$ with an arc of a circle having radius $\boldsymbol{s}$ in such a way that, at points where an arc adjoins a line segment, the tangent from the arc matches the 
tangent from the adjoining segment. It can then be calculated that the mean curvature of the arc near $f(i)$ equals the radian measure of the angle $\boldsymbol{\sigma}_{i}$ between $f^{\prime}\left(t t^{*}\right)$ and $f f^{\prime}\left(\left(t t_{i} \sim\right)\right.$, where the notation $f^{\prime}(t+)$ and $f^{\prime}\left(t_{i}\right)$ has been used to denote the limit of $f^{\prime}(t)$ as $t$ approaches $t_{i}$, from the right and left respectively. Since the line segments contribute nothing to the mean curvature and $\boldsymbol{\sigma}_{i}=\cos ^{-1}(f(t+) . f(t \sim))$ the following definition is obtained:

$$
M(f)=M\left({ }_{i=2}^{n-1} f_{\mathfrak{f}}\right)_{i=2}^{n-1}=\wedge \mathbf{0}_{i}=\mathrm{X} ! \cos ^{1}(f(t t) \cdot f(t i)) \text { (D.3) }
$$

Although we defined $M$ as an integral, for piece-wise linear segments it reduces to the sum of angles metric used by others $[6-9,11]$; our contribution is to identify the sum of angles as a limiting case of the definition given via the integral.

\section{Appendix E}

Let it be assumed that there is a sequence of points $x_{1}, x_{2}, \ldots . x n$ in $\boldsymbol{R}^{d}$, which are close to some idealized curve, and assume positive radii $r_{l}, r_{2}, \ldots, r_{n}$ with the interpretation that for each $i$, there exists a point on the idealized curve whose distance to $x i$ is less than or equal to $r i$. In other words, it is assumed that the idealized curve passes through each 'data ball', $B_{i}$, having center $x i$ and radius $r i$. A function $f:[a, b]-\gg \boldsymbol{R}^{d}$ is said to be a path (sequentially) through the data balls $\mathrm{B}_{\mathrm{i}}$ if there exist $a=t_{1}<\ldots<t n=\mathrm{b}$, such that $f(t i)$ e $B i$ for $i=1,2, \ldots, n$.

We assume that the data balls have empty intersection (i.e., there is no point common to all the data balls), and consequently it can be shown that there exists a unique shortest path through the data balls denoted by:

$$
f_{1}:[0, L 1] \rightarrow R^{d},
$$


where $L_{1}$ denotes the length of the shortest path. The numerical computation of the shortest path $f_{l}$ will be discussed in Appendix F; the shortest path $f_{l}$ is a unit speed piecewise linear function, as described in equation D.1, satisfying:

$$
f l\left(t_{i}\right) G B i \quad \text { for } i=1,2, \ldots \ldots, n \text {. }
$$

The shortest path is used to define the mean curvature:

$$
M(\{B i\})=M(f 1)
$$

which is defined by $D .3$, since $f_{1}$ is a unit speed piecewise linear function.

There remains the problem of defining $J(\{B i\})$ and $K(\{B i\})$. The shortest path $f_{l}$ is known to satisfy $E .1$, where the values $0=t 1<t_{2}<\ldots<t n=L_{1}$ are known; the numerical computation of $f_{1}$ provides these values. The following optimization problem has been studied by Kersey [15] and is expected to have a unique solution under the assumption that there does not exist a straight line that passes sequentially through the data balls $\left\{B_{\mathrm{i}}\right\}$.

Optimization Problem: For twice continuously differentiable functions $f_{2}:\left[0, L_{1}\right] \rightarrow \boldsymbol{R}^{d}$, minimize:

$$
\text { Ji) } \quad \tilde{2}^{L_{1}} \tilde{u}^{2}(t) d t
$$

subject to the near interpolation constraints $f_{2}$ (ti) sBi for $i=1,2, \ldots, n$.

The function $f_{2}$, which solves this optimization problem turns out to be a parametric natural cubic spline and can be found using an algorithm proposed by Kersey [16]. The curve $f_{2}$ is not a unit speed curve; however, the choice of the $\left\{t_{i}\right\}$ in conjunction with the near 
interpolation constraints are designed to keep $f_{2}$ close to unit speed. With $L_{2}$ denoting the length of this curve (the smoothest curve):

$$
L_{2}=I^{1} f_{2}^{\prime}(t) d t
$$

let $f_{2}:\left[0, L_{2}\right] \rightarrow \boldsymbol{R}^{d}$ be the unit speed reparameterization of $f_{2}$, namely

$$
f_{2}(s)=f_{2}(t), \text { where } s=1 t f_{2}^{\prime}(\mathrm{r}) d r
$$

Finally, the definitions are made:

$$
J(\{B i\})=J\left(f_{2}\right) \text { and } K(\{B i\})=K\left(f_{2}\right) .
$$

\section{Appendix F}

In this section we present an overview of the algorithm used for computing the shortest path through the data balls $\{B i\}$. The algorithm is a variation on an algorithm proposed by Kersey [16], and uses a least squares approach.

Problem F.1. Given time values $0=t_{1}<t_{2}<\ldots<t_{n}$ and positive weights $w_{1}, w_{2}, \ldots, w n$ ,related to the data ball sizes, find the path $f:\left[t_{1}, t_{n} \longrightarrow \rightarrow \boldsymbol{R}^{d}\right.$ which minimizes:

$$
\left|n f^{\prime}(t)\right|^{2} \quad d t \quad+Y_{i=1} w_{i} f\left(t_{i}\right)-x_{i} \quad 2
$$

This optimization problem is well understood and the path can be efficiently found by solving a banded system of linear equations. The solution, $f$, is a continuous piece-wise linear path, but is not necessarily unit speed. If the problem F.1 is repeatedly solved, while changing the weights, it is possible to approximately solve the non-linear optimization problem:

Problem F.2 Given time values $0=t_{1}<t_{2}<\ldots<t_{n}$, find the path $f:\left[t_{1}, t_{n}\right]-\gg f^{d}$ which minimizes: 


$$
|f(t)| d t
$$

$t 1$

subject to the constraints $f(t i) \quad B_{i}, i=1,2, \ldots, n$.

For this, the weight update suggested by Kersey [16] is recommended:

$$
w=\frac{|f(t)-i|}{r i} i_{w}{ }^{\wedge} \quad i=2,3, \ldots, n
$$

where the weight $w_{i}$ on the right is the previous weight and the weight $w_{i}$, on the left is the updated weight. Note that the objective function in problem F.2 is not the length of the path $f$ because $f(t) \backslash$ has been squared. However, if $f$ were unit speed, then $f(t) \backslash$ would equal $|f(t)|=1$ and so the objective function would equal the length. This can be obtained iteratively by repeatedly solving problem F. 2 while updating the time values according to $t_{1}=$ 0 and

$$
t i=t_{i-1}+|f(\sim)-f(\sim !)|, i=2,3, \ldots, n
$$

where the previous time values have been denoted $\{\sim t\}$. Thus the problem can be solved iteratively:

Problem F.3. Find the shortest unit speed path $f$ which satisfies, for some choice of time values $0=t I<t_{2}<\ldots . .<t$, the constraints:

$$
f\left(t_{i}\right) \quad \text { Bi } \quad i=1,2, \ldots . . n .
$$

There is no theoretical proof that the algorithm works; however, several thousand shortest path problems have been solved during the course of the present series of experiments, and it has converged in all cases. It has also been adapted to handle the case where the data balls $B i$ are ellipses or rectangular boxes instead of balls, and no difficulties have been experienced with these cases. 


\section{Appendix G}

Brownian motion with variance $\sigma^{2}$ can be defined as $\mathrm{B}(\mathrm{t})=\mathrm{B}(0)+\sigma \quad W(s) d s$, where $\mathrm{B}(0)$ is the initial value and $\mathrm{W}$ is standard white noise with a variance of unity. If $0=t_{0<} t_{1 \ldots<<} t_{n \text {, }}$ then we can synthesize Brownian motion as $\mathrm{B}\left(t_{\mathrm{i}}\right)=\mathrm{B}\left(t_{i-1}\right)+\left(t_{i}-t_{i-1}\right) . \sigma \mathrm{N}$ where $\mathrm{N}$ denotes a random number with a normal distribution. Brownian motion is continuous, with probability unity, but is not differentiable.

We can define integrated Brownian motion with variance $\sigma^{2}$ by $x(t)=x(0)+{ }_{0}^{t} B(s) d s$ where B is Brownian motion defined above. Integrated Brownian motion is continuously differentiable which is why we prefer it over Brownian motion. Integrated Brownian motion can be defined at times $0=t_{0<} t_{1 \ldots<<} t_{n,}$ as follows:

1. $x^{\prime}\left(t_{0}\right)=$ pnescribed initial slope,

2. $x\left(t_{0}\right)=$ prescribed initial position,

3. For $i=1,2, \ldots, n$ do:

$$
\begin{aligned}
& x^{\prime}\left(t_{i}\right)=x^{\prime}\left(t_{i-1}\right)+\sqrt{\Delta l_{i}} \sigma N \\
& x\left(t_{i}\right)=x\left(t_{i-1}\right)+\left(x^{\prime}\left(t_{i-1}\right)+J^{\prime}\left(l_{i}\right)\right) \frac{\Delta l_{i}}{2}+\sqrt{\frac{\Delta l_{i}^{3}}{12}} \sigma N,
\end{aligned}
$$

where $\Delta t_{\mathrm{i}}=t_{i}-t_{i-1}$

A parametric curve, with unit speed at all times, based on integrated Brownian motion can be synthesized by

1. $f^{\prime}\left(t_{0}\right)=$ prescribed inilial tunil tangent vector (L.e. $\left\|f^{\prime}\left(t_{0}\right)\right\|=1$ is required).

2. $f\left(L_{0}\right)=$ prescribed initial position

3. For $i=1,2, \ldots, n$ do

$$
\begin{aligned}
f^{\prime}\left(t_{i}\right) & =f^{\prime}\left(t_{i-1}\right)+\sqrt{\Delta t_{i}}\left(\sigma_{1} N, \sigma_{2} N, \sigma_{3} N\right) \\
f^{\prime}\left(t_{i}\right) & =f^{\prime}\left(t_{i}\right) /\left\|f^{\prime}\left(t_{i}\right)\right\| \\
& \text { apply restraint, if any } \\
f\left(t_{i}\right) & =f\left(t_{i-1}\right)+\left(f^{\prime}\left(t_{i-1}\right)+f^{\prime}\left(t_{i}\right)\right) \frac{\Delta t_{i}}{2}+\sqrt{\Delta t_{i}^{3} / 12}\left(\sigma_{1} N, \sigma_{2} N, \sigma_{3} N\right)
\end{aligned}
$$

In our experiments, the above-mentioned restraints are actually constraints on the unit tangent vector $f^{\prime}(t)$. If we want the synthesized curve to be loosely tied to the $z$-axis, then we require that 
the angle between $f^{\prime}(t)$ and the $x y$-plane be no less than some prescribed angle ; this is enforced by rotating $f^{\prime}\left(t_{i}\right)$ toward the $z$-axis by the smallest angle needed to satisfy the constraint. Note that the curve is more strongly tied to the $z$-axis as $\varphi$ approaches $90^{\circ}$. Alternatively, if we want the synthesized curve to be loosely tied to the $x y$-plane, then we prevent the angle between $f^{\prime}\left(t_{i}\right)$ and the $x y$-plane from exceeding some prescribed angle.

For curves $C$ which are loosely tied to the $z$-axis, we consider the task of approximating the values of $J$ and $K$, given only two planar projections of $C$; namely the projections of $C$ onto the $x z$ and $y z$ planes, denoted $C_{x z}$ and $C_{x z}$ respectively. For this purpose, let us assume that the three curves $C$, $C_{x z}$ and $C_{y z}$ have unit speed parameterizations

$$
\begin{array}{ll}
C_{x *}: & \left(f_{1}(t), f_{2}(t), f_{3}(t)\right), \quad 0 \leq t \leq L \\
C_{x z}: & \left(g_{1}(t), 0_{1} g_{3}(t)\right), \quad 0 \leq t \leq L_{x x} \\
C_{y z}: & \left(0, h_{2}(t), h_{3}(t)\right), \quad 0 \leq t \leq L_{y z t}
\end{array}
$$

Where $g_{1}, g_{3}, L_{x y}$ and $h_{1}, h_{3}, L_{y z}$ are known, but $f_{1}, f_{2}, f_{3}, L$ are unknown. Our approximation to $L$ is simply the average:

$$
\widetilde{L}=\frac{1}{2}\left(L_{x z}+L_{y z}\right)
$$

Our approximation to the unknown quantity $J$, which can be written as

$$
\left.\left.J=\int_{0}^{L}: f_{1}^{\prime \prime}(t)\right]^{2} d t+\int_{0}^{L} f_{2}^{\prime \prime}(t)\right]^{\overline{2}} d t+\int_{0}^{L}\left[f_{3}^{\prime \prime}(t)_{j}^{2} d t\right.
$$

Is the following:

$$
\widetilde{J}=\int_{0}^{L_{x x}}\left[g_{1}^{\prime \prime}(t)\right]^{2} d t+\int_{0}^{L_{k x}}\left[h_{2}^{\prime \prime}(t)\right]^{2} d t+\frac{1}{2}\left(\int_{0}^{L_{x x}}\left[g_{3}^{\prime \prime}(t)\right]^{2} d t+\int_{0}^{L_{k x}}\left[h_{3}^{\prime \prime}(t)\right]^{2} d t\right) .
$$

This is motivated by the recognition that the first term in $J$ is approximated by the first term in ${ }^{J}$, and similarly for the second term; while the third term in $J$ has two approximations, which we have averaged to form the third term in ${ }^{J}$. Finally, our approximation to $K=\mathbf{V}(L J)$ is simply

$$
\widetilde{\boldsymbol{K}}=\sqrt{\tilde{L} \tilde{J}}
$$


In order to test the accuracy of these approximations, for each angle ( $\mathbf{p}$ in $\{850,800,750, \ldots$, $450\}$ we synthesized 1000 parametric curves, which are loosely tied to the $z$-axis by angle $\mathbf{q}$, as described above, and computed the relative distance between $\boldsymbol{L} . . . J . K$ and $L, J, K$

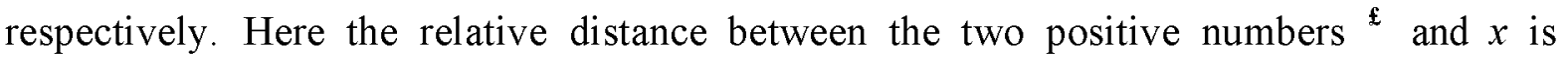
defined by

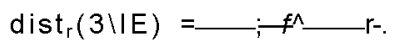




\section{References}

[1] Caro CG, Fitz-Gerald JM, Shroter RC. Artheroma and arterial wall shear. Observation, correlation and proposal of a shear dependent mass transfer mechanism for artherogenesis. Proc Roy Soc Lond B 1971; 177: 109-59.

[2] Friedman MH, Deters OJ, Mark FF, Bargeron CB, Hutchins GM. Arterial geometry affects haemodynamics: a potential risk factor for atherosclerosis. Atherosclerosis $1983 ; 46: 225-31$.

[3] Capowski JJ, Kylstra JA, Freedman SF. A numeric index based on spatial frequency for the tortuosity of retinal vessels and its application to plus disease in retinopathy of prematurity. Retina 1995; 15:490-500.

[4] Smedby Ö, Högman N, Nilsson S, Erikson U, Olsson AG, Walldius G. Twodimensional tortuosity of the superficial femoral artery in early atherosclerosis. J Vasc Res 1993;30: 181-91.

[5] Lotmar W, Freiburghaus A, Bracher D. Measurement of vessel tortuosity on fundus photographs. Graefe's Arch Clin Exp Ophthalmol 1979; 211: 49-57.

[6] Kimball B, Bui S, Dafopoulos N. Angiographic features associated with acute coronary artery occlusion during elective angioplasty. Can J Cardiol 1990; 6: 327-32.

[7] Brinkman AM, Baker PB, Newman WP, Vigorito R, Friedman MH. Variability of human coronary artery geometry: an angiographic study of the left anterior descending arteries of 30 autopsy hearts. Ann Biomed Eng 1994; 22: 34-44.

[8] Hart WE, Goldbaum M, Côté B, Kube P, Nelson MR. Measurements and classification of retinal vascular tortuosity. Intl J Med Informatics 1999; 53: 239-52. 
[9] Bullitt E, Gerig G, Pizer SM, Lin W, Aylward SR. Measuring tortuosity of the intracerebral vasculature from MRA images. IEEE Trans Med Imag 2003; 22: 1163 71.

[10] Dougherty G and Varro J. A quantitative index for the measurement of the tortuosity of blood vessels. Med Eng Phys 2000; 22: 567-74.

[11] Grisan E, Forrachia M, Ruggeri A. A novel method for the automatic evaluation of retinal vessel tortuosity. Proc Ann Int Conf, IEEE Eng Med Biol Soc, Sept 2003, Cancun, Mexico.

[12] Crenshaw HC, Ciampaglio CN, McHenry M. Analysis of the three-dimensional trajectories of organisms: estimates of velocity, curvature and torsion from positional information. J Exp Biol 2000; 203: 961-82.

[13] Saidléar CA. Implementation of a quantitative index for 3-D arterial tortuosity. M.Sc. thesis, University of Dublin, 2002.

[14] Dapunt OE, De Asha RA, Griepp EB, Midulla PS, Griepp RB. Computer-generated 3D representations of the aorta: a new tool in the management of aortic aneurysm patients. Thoracic Cardiovasc Surg 1994; 42: 25-8.

[15] Kersey SN. Best near interpolation by curves: existence. Siam J Numerical Analysis 2000; 38: 1666-75.

[16] Kersey SN. On the problems of smoothing and near interpolation. Mathematics of Computation 2003; 72: 1873-85.

[17] Thomas GB and Finney RL. 'Calculus and Analytic Geometry'; $8^{\text {th }}$ edition; AddisonWesley, 1992, p.732-8.

[18] Horowitz P, Hill W. 'The art of electronics'; $2^{\text {nd }}$ edition; Cambridge University Press, Cambridge, 1989, ch.9. 
[19] Bluemka DA, Stillman AE, Bis KG, Grist TM, Baum RA, D'Agostino R, Maiden ES, Pierro JA and Yukel EK. Carotid MR angiography: phase II study of safety and efficacy for MS-325. Radiology 2001; 219: 114-22.

[20] Lamarque F, Le Gargasson JF and Gautier V. New confocal scanning-laser ophthalmoscope device: double pass in a fiber optic. Proc. SPIE 1999; 3570: 256-62.

[21] Goatman K, Manivannan A, Hipwell JH, Sharp PF, Lois N and Forrester JV. Automatic registration and averaging of ophthalmic autofluorescence images. Poster Session 2, Medical Image Understanding and Analysis, 2001, University of Birmingham, England.

[22] Bidiwala SB, Mansour MS, Stengel CK, Klein SA, Carroll SM, Koenig SC, Desoky AH, Tobin GR, Maldonado C, Barker JH. Quantification of the morphological features of a full microvascular network. Med Biol Eng Comput 1998; 36: 621-6. 
Table 1

Tortuosity metrics for simple helices. (a, $\mathrm{b}$ and $\mathrm{L}$ have dimensions of length, $\mathrm{x}$ has dimensions of inverse length, and $M$, $J$ and $K$ are dimensionless).

\begin{tabular}{|c|c|c|c|c|c|c|c|c|}
\hline $\begin{array}{l}\text { Radius } \\
\mathrm{a}\end{array}$ & $\begin{array}{l}\text { Pitch } \\
\text { b }\end{array}$ & $\begin{array}{c}\text { No. of turns } \\
\mathrm{N}\end{array}$ & $\begin{array}{l}\text { Theoretical } \\
\text { curvature, } \mathrm{x}\end{array}$ & Data set & $\begin{array}{l}\text { Length, } \\
\quad L\end{array}$ & $\begin{array}{c}\text { Mean curvature, } \\
M\end{array}$ & $\begin{array}{l}\text { Mean square } \\
\text { curvature, } J\end{array}$ & $\begin{array}{c}\text { rms } \\
\text { curvature }\end{array}$ \\
\hline 10 & 0 & 1 & 0.1 & $3-\mathrm{D}$ & 62.8 & 6.22 & 0.624 & 6.26 \\
\hline \multirow[t]{3}{*}{10} & 10 & 1 & 0.05 & 3-D & 88.8 & 4.40 & 0.221 & 4.43 \\
\hline & & & & coronal & 76.4 & 3.14 & 0.207 & 3.98 \\
\hline & & & & sagittal & 76.4 & 3.08 & 0.203 & 3.94 \\
\hline \multirow[t]{3}{*}{10} & 20 & 1 & 0.02 & 3-D & 140 & 2.78 & 0.056 & 2.80 \\
\hline & & & & coronal & 133 & 1.85 & 0.034 & 2.14 \\
\hline & & & & sagittal & 133 & 1.82 & 0.034 & 2.12 \\
\hline \multirow[t]{3}{*}{10} & 40 & 1 & 0.006 & 3-D & 259 & 1.51 & 0.009 & 1.52 \\
\hline & & & & coronal & 255 & 0.98 & 0.005 & 1.10 \\
\hline & & & & sagittal & 255 & 0.98 & 0.005 & 1.10 \\
\hline \multirow[t]{3}{*}{10} & 100 & 1 & 0.0009 & 3-D & 631 & 0.62 & 0.001 & 0.62 \\
\hline & & & & coronal & 630 & 0.04 & 0.0003 & 0.44 \\
\hline & & & & sagittal & 630 & 0.04 & 0.0003 & 0.44 \\
\hline \multirow[t]{3}{*}{10} & 10 & 2 & 0.05 & $3-\mathrm{D}$ & 178 & 8.84 & 0.443 & 8.87 \\
\hline & & & & coronal & 153 & 6.28 & 0.414 & 7.96 \\
\hline & & & & sagittal & 153 & 6.22 & 0.411 & 7.92 \\
\hline \multirow[t]{3}{*}{20} & 20 & 1 & 0.025 & 3-D & 178 & 4.40 & 0.110 & 4.43 \\
\hline & & & & coronal & 153 & 3.14 & 0.104 & 3.98 \\
\hline & & & & sagittal & 153 & 3.08 & 0.102 & 3.94 \\
\hline 10 & $(10,20,40)$ & 3 & 0.0253 & 3-D & 488 & 8.69 & 0.285 & 8.75 \\
\hline \multirow[t]{3}{*}{10} & 17.18 & 1 & 0.0253 & $3-\mathrm{D}$ & 125 & 3.13 & 0.079 & 3.15 \\
\hline & & & & coronal & 117 & 2.11 & 0.052 & 2.46 \\
\hline & & & & sagittal & 117 & 2.07 & 0.051 & 2.44 \\
\hline
\end{tabular}


Table 2

For each angle ( $\mathrm{p}$ in $\{850,800,750, \ldots, 450\} 1000$ parametric curves, which are loosely tied to the z-axis by angle ( $\mathrm{p}$, have been simulated, and for each curve, the values of $\mathrm{L}, J$, $K$ are computed and compared, in terms of relative distance, with their corresponding approximations $L, J, K$ which are computed from two planar projections of the curve (see Appendix $\mathrm{G}$ for details). The relative distance between two positive numbers ${ }^{\mathrm{s}}$ and $\mathrm{x}$, $\operatorname{dist}_{\mathrm{r}}\left({ }^{5}, \mathrm{x}\right)$ is defined in equation G.5: the values reported here correspond to the 95-th percentile of the 1000 trials. In other words, 950 of the 1000 computed relative distances fall at or below the listed value.

$\begin{array}{lccc}\text { A } & <\operatorname{list}_{\mathrm{r}}\left(\mathrm{L}_{1} L\right) & \operatorname{diat}_{\mathrm{T}}(\mathrm{JH} J) & \text { dist }_{r}(K, K) \\ 85 & 0,001186 & 0.002533 & 0.0007075 \\ \text { M1 } & 0.005103 & 0.01167 & 0.003435 \\ 75 & 0.01259 & 0.03262 & 0.0105 \\ \text { VII } & 0 . \mathrm{D} 234 \mathrm{~S} & 0.066 \mathrm{~S} 9 & 0.02217 \\ 65 & 0.03 \mathrm{~S} 02 & 0.1205 & 0.04114 \\ 60 & 0.05736 & 0.1877 & 0.06189 \\ \text { r.r. } & 0.0 \mathrm{OS} 176 & 0.302 & 0.1011 \\ \text { r.ii } & 0.1081 & 0.435 & 0.1417 \\ \text { sir, } & 0.1412 & 0.6906 & 0.2252\end{array}$


Abbreviated title: A robust measure of vascular tortuosity 


\section{Figure Legends}

Fig. 1 A helix with added random noise $(\mathrm{SNR}=34 \mathrm{~dB})$.

Fig.2 Values of (a) $M$ and (b) $K$ for noisy helices as a function of SNR using different data ball sizes, $r$.

Fig.3 Synthesized blood vessel (dashed lines) and the smoothest path through it (solid line) using a data ball size, $r$, equal to (a) the local radius of the vessel $\left(\mathrm{R}_{\mathrm{i}}\right)$ and (b) onequarter of the local radius of the vessel $\left(\mathrm{R}_{\mathrm{i}} / 4\right)$.

Fig.4 Values of $K$ (top) and $M$ (bottom), shown using dashed lines, as a function of resolution (pixel size, $h$ ) for a synthesized vessel of nominal radius, $R_{\text {nom }}=0.07$ : the data ball sizes, $r$, used were (a) $\mathrm{R}_{\mathrm{i}} / 10$ (b) $\mathrm{R}_{\mathrm{i}} / 4$ and (c) $\mathrm{R}_{\mathrm{i}} / 2$. The dark solid lines indicate the zero-phase five-point running average values of $K$ and $M$. Horizontal lines show the $\pm 10 \%$ limits on the best $K$ (dotted lines) and $\mathrm{M}$ (dash-dot lines) values.

Fig. 5 Values of $K$ (top) and $M$ (bottom), shown using dashed lines, as a function of resolution (pixel size, $h$ ) for a synthesized vessel of nominal radius, $R_{\text {nom }}=0.07$ using $50 \%$ SPPR. (The data ball sizes, $r$, used were (a) $\mathrm{R}_{\mathrm{i}} / 10$ (b) $\mathrm{R}_{\mathrm{i}} / 4$ and (c) $\mathrm{R}_{\mathrm{i}} / 2$. The dark solid lines indicate the zero-phase five-point running average values of $K$ and $M$. Horizontal lines show the $\pm 10 \%$ limits on the best $K$ (dotted lines) and $M$ (dash-dot lines) values).

Fig.6 Values of the tortuosity metrics for type III abnormalities using (a) circles and (b) elliptic helices to model 2-D and 3-D behavior respectively. The solid lines indicate $K$, the dash-dot lines $J$, the dashed lines $M$, and the dotted lines the distance factor, DF. 